\title{
Comparative Study of the Accuracy of Analytical Inductance Formulae for Square Planar Spiral Inductors
}

\author{
H. A. Aebischer ${ }^{1 *}$ \\ ${ }^{1}$ LEGIC Identsystems AG, Switzerland \\ *corresponding author, E-mail: hubert. aebi scherelegic.com
}

\begin{abstract}
In the design of radio frequency (RF) microelectronic integrated circuits (IC's) and of antennas for short-wave radio frequency identification (RFID) and telemetry systems, planar spiral coils are important components. Many approximate analytical formulae for calculating the inductance of such coils can be found in the literature. They all approximate the direct current (DC) inductance. Its applicability to high frequencies depends on the ratio of the conductor width to the skin depth. In many cases, the DC inductance is a good approximation in RFID and telemetry systems at 13.56 MHZ, and in RF IC's up to about $4 \mathrm{GHz}$. The formulae can simplify the problem of designing inductors to a predefined inductance considerably. But the error statistics given by different authors cannot be compared because they are based on different or unknown domains of definition. Hence, it is not possible to decide which formula is best in a given case by merely studying the literature. This paper compares the maximum relative errors of six of some of the most cited formulae in the literature. Five of them are scalable, which means that they are valid for all coil sizes and all inductance ranges. To all formulae, the same domains of definition are applied. Each of them spans all four dimensions of the parameter space. Precise inductances are obtained numerically with the help of the free scientific and industrial standard software FastHenry2 and used as reference values to calculate the errors of the formulae. It has been found that the alleged maximum errors reported by some authors are far too optimistic. Only two formulae feature small enough errors to be useful in circuit design. The method and the domains of definition applied in the present study may also prove useful for the assessment of future formulae.
\end{abstract}

\section{Introduction}

The interest in planar spiral coils has been spurred by their application as inductors in radio frequency (RF) microelectronic integrated circuits (IC's) [1] and as antennas in shortwave radio frequency identification (RFID) [2] and telemetry systems [3]. In the design of inductors in RF IC's, the inductance needed is specified in the circuit diagram. In the case of RFID and telemetry antennas, it is constricted by design rules. In any case, the coil must be designed to a predefined inductance. This represents a reverse problem. It can only be solved indirectly, by calculating the inductance of many coils, and by choosing the one whose inductance matches the predefined value best. In doing so, some method must be used to calculate the inductance of a coil from its geometric design parameters, and the calculation must be repeated many times. In principle, this can be done with the help of numerical methods, e.g. as implemented in freeware [4]. But even for a single inductor design, creating the input data file required by the numerical software to define the design is tedious, particularly if the coil has many windings, let alone if the calculation must be repeated for many different designs, as is the case in a reverse problem. Besides, the computer run time may be long. Hence, this way of solution is impractical.

Reverse problems can be solved much faster and easier when an analytical inductance formula is available. The Greenhouse method [5] allows precise calculations of coil inductance at direct current (DC) using analytical formulae. It consists of dividing the coil into its constituent straight conductor segments and calculating their partial selfinductance and all mutual inductances between them separately using analytical formulae, and summing up all the contributions. For larger numbers of turns, the calculation gets tedious because the method doesn't provide an analytical formula that explicitly depends on the design parameters, like e.g. the number of turns, the winding distance, etc. This makes the method impractical for designing a coil to a predefined inductance. Therefore, many researchers have worked on finding approximate analytical formulae that explicitly depend on the design parameters. Six of the most cited ones, according to Google Scholar, are compared in this study.

Crols et al. ([6], Table 1) presented an empirical formula that is mainly based on the ratio of the coil area covered by metal to the total area.

Ronkainen et al. ([7], equation (1)) published an empirical formula that depends on the product of an exponential function and a power function of various ratios of the design parameters.

Mohan et al. ([8], equations (1) - (3)) presented three formulae. They included a comprehensive Table listing 61 coil designs and the respective errors obtained in comparison to measurements ([8], Table IV). The first formula, termed "modified Wheeler formula", was obtained by modifying 
an empirical formula known from the literature. The second formula, by contrast, is based on physical principles, namely, on a current sheet approximation. The third formula is empirical again. It is a product of power functions of geometric coil parameters, which they termed a "monomial expression".

Jenei et al. ([9], equation (7)) presented a formula based on physical principles. It represents an approximation to the Greenhouse method [5].

All these formulae seem to have been set up for DC. In any case, none of them contains the frequency as a parameter. For coils used in RF IC's, where the conductor width is of the order of a few microns, the DC inductance is a good approximation at frequencies up to the $\mathrm{GHz}$ range, because the skin depth for copper only drops to below one micron just above $4 \mathrm{GHz}$. The DC inductance also applies to many coils used as RFID and telemetry antennas. Although they are larger than those used in IC's, this is compensated to some extent by the lower frequency of $13.56 \mathrm{MHz}$.

The relative error of a formula is calculated by taking the modulus of the relative deviation of the inductance obtained by the formula from a precise reference value calculated numerically. For simplicity, throughout the paper, the maximum relative error of a formula over some domain of definition is called its maximum error over that domain.

It will be shown that some of the alleged maximum errors of the formulae reported in the literature are far too optimistic. Besides, a given error specification can almost never be compared with another one indicated for a different formula. This is because the error statistics is either based on different domains of definition, or the domain of definition in one or both cases is not even known. Modifying the domain of definition can change the maximum error of a formula considerably, as the results of the present study attest. So, it is difficult for the design engineer to decide which formula to use, let alone to estimate the error to be expected in the case at hand. Most importantly, for a design engineer, only the maximum error over a specified domain of definition is meaningful. Any other error indication, like e.g. mean absolute percentage error, relative root mean square (RMS) error etc., says absolutely nothing about the upper limit that the error is guaranteed not to exceed in any case at hand.

Thus, it would be helpful to have reliable and standardized accuracy data of the maximum error of these formulae over a specified domain of definition. The present paper provides this data. Besides, it presents a consistent exposition of all design parameters used in the six formulae to describe square planar spiral inductors. It is shown that transforming the absolute or dimensional design parameters into relative or dimensionless ones allows to reduce the parameter space dimension from five to four. Further, two equations are derived allowing to decide whether a given combination of parameter values represents a geometrically well-defined coil or not, one for dimensional parameters, and the other for dimensionless ones. For the error analysis, the paper defines four domains of definition for the dimensionless parameters which guarantee that, firstly, only geometrically valid combinations of values are considered, and secondly, that all four dimensions of the parameter space are scanned. This last point is essential because, if not all independent parameters are varied, then the analysis is likely to miss the true maximum error. The negligence of this very aspect has invalidated some of the error analyses found in the literature.

Some of the formulae only apply to square coils, and others only to such of integer number of turns. Hence, this study is restricted to square coils of integer number of turns. Further, all six formulae assume a rectangular conductor cross section. Originally, they were all derived for designing inductors in RF IC's, whereas in this study, their fitness for designing RFID and telemetry antennas is also tested.

Section 2 comprehensively explains all the parameters needed to describe square planar spiral coils. It derives the parameter transformations and the equations embodying a validity test for a given combination of parameter values. Section 3 presents the method used for the error analysis, specifies the domains of definition, and lists the sampling values chosen for the dimensionless parameters. Section 4 discusses the six analytical formulae, and it presents the results of the error analysis. Section 5 closes the paper with some conclusions.

\section{Description of the coils}

Fig. 1 shows the layout of a square planar spiral coil with three windings, together with the geometric definition of the absolute or dimensional design parameters. Redundant parameters are used deliberately. This is necessary to be able to present and discuss the inductance formulae in a consistent manner. These parameters are:

- $\quad N$, number of turns or windings, $N \geq 2$.

- $A_{o}$, outermost side length.

- $A$, outermost mid-conductor side length.

- $A_{i}$, innermost mid-conductor side length.

- $A_{i i}$, innermost side length.

- $\quad w$, winding distance or -pitch.

- $g$, gap or spacing between windings.

- $s$, conductor width.

- $h$, conductor thickness or height (hidden in Fig. 1).

The following relations hold, as can easily be verified with the help of Fig. 1:

$$
\begin{gathered}
A_{o}=A+s . \\
A_{i}=A-2(N-1) w . \\
A_{i i}=A_{i}-s . \\
w=s+g .
\end{gathered}
$$

Various sets of five independent parameters can be used to describe a coil, e.g.

$$
\{N, A, w, s, h\} \text {. }
$$




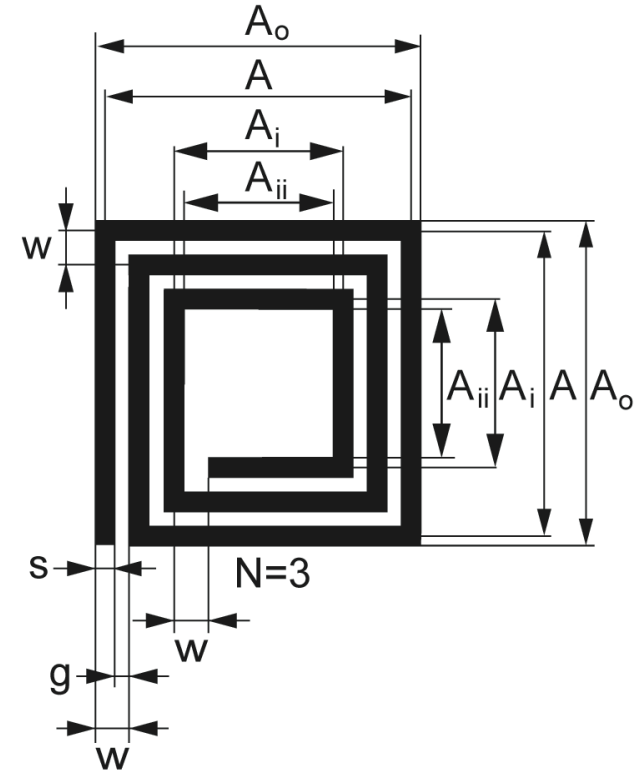

Figure 1: The layout of a square planar spiral coil of three windings $(N=3)$ with its design parameters.

Coil inductance scales linearly with size, to a very good approximation. This means that, if all four size-dependent parameters (e.g. $A, w, s$, and $h$ ) are multiplied by the same factor, then coil inductance multiplies by the same factor. This can be seen by inspecting the formulae for the partial self-inductance of single straight conductors and for the mutual inductance $M$ between such, which are both very similar (see e.g. [10]). The formula for $M$ can be put into the form

$$
M \approx \frac{\mu_{0}}{2 \pi}\left[l \cdot \log \left(\frac{\sqrt{l^{2}+d^{2}}+l}{d}\right)-\sqrt{l^{2}+d^{2}}+d\right],
$$

where $l$ is the conductor length, $d$ is the distance between the longitudinal axes of two parallel conductors, and $\mu_{0}$ is the permeability of the vacuum (see section 4 ). The square roots can be rewritten in the form $l \cdot \sqrt{1+\lambda^{2}}$, where $\lambda=$ $d / l$. It follows that the square root scales exactly linearly with size since $\lambda$ as a ratio of two size-dependent quantities is itself size-independent. Consequently, the whole argument of the logarithm in the expression above is a ratio of two size-dependent terms and therefore size-independent. Hence, the whole expression above scales exactly linearly with size. And coil inductance is nothing but a linear combination of such partial self- and mutual inductances. The reason why, nevertheless, coil inductance only scales linearly to a very good approximation is that the above expression is only an approximation to a six-dimensional integral, albeit a very good one, see [10]. Now, if an analytical coil inductance formula scales linearly with size, then it is said to be scalable. Consequently, the relative error of a scalable formula doesn't depend on the absolute coil size, but only on any three mutual ratios of four size-dependent parameters, like e.g. $A / w, w / s, s / h$, and their reciprocals. So, the number of parameters can be reduced from five to four: the number of windings $N$ plus three size-independent ratios. The size of the coil is then defined by any size-dependent parameter.

Instead of one of the possible ratios containing $A$, like e.g. $A / w, A / s, A / h$, and their reciprocals, a different parameter that depends on these ratios, but is more useful in the error analysis, is used here as the first dimensionless parameter, namely, the filling factor $\rho$. It is defined as

$$
0<\rho=\frac{A_{o}-A_{i i}}{A_{o}+A_{i i}}<1,
$$

where $A_{o}$ is given by equation (1), and $A_{i i}$ by equations (2) and (3). The filling factor is a normalized measure of the extent to which the area taken by the coil is used up or covered by its windings. It is helpful to derive an alternative equation for $\rho$ that directly depends on the parameters (5). To this end, equations (1) - (3) are substituted into (6), yielding

$$
\rho=\frac{(N-1) w+s}{A-(N-1) w}
$$

The next two dimensionless parameters used here are the relative winding distance $\kappa$ defined as

$$
\kappa=\frac{w}{s}>1
$$

and the cross-sectional aspect ratio $\gamma$,

$$
\gamma=\frac{s}{h} \geq 1 \text {. }
$$

The condition $\gamma \geq 1$ reflects a usual constraint on printed circuit boards (PCB's) and in IC's. Together with $N$, the result is a set of four dimensionless parameters,

$$
\{N, \rho, \kappa, \gamma\}
$$

Equations (7) - (9) allow to transform the absolute or dimensional design parameters (5) into the relative or dimensionless ones (10). As is shown below, for any set of values of the dimensionless parameters (10), the corresponding dimensional ones $w, s$, and $h$ are proportional to the side length $A$. Since the inductance scales linearly with size, in the error analysis based on parameters (10), the value of $A$ is irrelevant, provided the examined inductance formula is scalable.

In the error analysis, the reference designs will be described by combinations of values of the dimensionless parameters (10) to benefit from the reduction of the parameter space dimension. The inverse transformation will be needed to transform these values back into dimensional ones (5). For the side length $A$ some fixed, standardized value may be chosen. The resulting dimensional parameters will then be used as input for the numerical software to calculate the precise reference inductances, see section 3 . To derive the 
equations for the inverse transformation, we need the relative conductor width $\eta$ (as an auxiliary parameter):

$$
\eta=\frac{S}{A} \ll 1
$$

The length $A$ of the outermost conductor segments of a spiral coil is usually much larger than the width $s$, so that $\eta \ll 1$. Substituting $s$ in equation (7) by

$$
s=\eta A
$$

and similarly, $w$ by

$$
w=\kappa \eta A,
$$

which follows from equations (8) and (12), and solving the equation that thus results from equation (7) for $\eta$, we find

$$
\eta=\frac{\rho}{(N-1)(1+\rho) \kappa+1} \ll 1 .
$$

Finally, it follows from equation (9) that $h$ is given by

$$
h=\frac{s}{\gamma} .
$$

Now, given the side length $A$, equations (12) - (15) allow to transform the dimensionless parameters (10) back into the dimensional ones (5). Equations (12), (13), and (15) confirm that $s, w$, and $h$ thus found by the inverse transformation are indeed proportional to the side length $A$.

It is important to note that it is not possible to freely choose any combinations of values for the design parameters. The reason is that, for some combinations, the last innermost conductor segment when spiraling inwards, whose length is $A_{i}-w$, see Fig. 1, may vanish completely, or even turn negative, so that the innermost turn is either incomplete or even ill-defined. General conditions must be found to avoid such a situation. Hence, the last innermost conductor segment must not be allowed to vanish. Let's say that its length $A_{i}-w$ should be at least $w$ or

$$
A_{i} \geq 2 w
$$

Equation (2) shows that this is equivalent to

$$
w \leq \frac{A}{2 N}
$$

This is the condition to test whether a design defined by a given combination of dimensional parameter values is geometrically valid or not. With the help of equations (13) and (14), it can be reformulated in terms of dimensionless parameters as

$$
\frac{1}{2 N}-\frac{\kappa \rho}{(N-1)(1+\rho) \kappa+1} \geq 0
$$

Only value combinations of $N, \rho$, and $\kappa$ satisfying this condition are valid. Reducing this expression to a common denominator, equating the nominator to zero and collecting terms in $\rho$, we get

$$
-(N+1) \kappa \rho+(N-1) \kappa+1=0 .
$$

We can easily find the root of this linear function in $\rho$. Since this involves a sign reversal, the final inequality for $\rho$ also reverses, and equation (18) is equivalent to

$$
\rho \leq \frac{(N-1) \kappa+1}{(N+1) \kappa} \text {. }
$$

In the limit $\kappa \rightarrow 1$, this results in

$$
\rho \leq \frac{N}{N+1}
$$

and in the limit $\kappa \rightarrow \infty$, we find

$$
\rho \leq \frac{N-1}{N+1}
$$

Equations (20) show that the largest value of $\kappa$ leads to the most stringent condition, i.e. to the lowest upper limit of $\rho$, and that the limit increases with increasing $N$. Hence, if equation (19) is observed for the lowest $N$ and the largest $\kappa$, then condition (18) is always fulfilled.

Since coil inductance scales linearly with size, one of the size-dependent parameters, e.g. $A$, can be made a linear factor in an analytical inductance formula, assuring its scalability. The remaining part of the formula can then be expressed in terms of the four size-independent parameters (10). Hence, the reduction of the parameter space dimension is not restricted to error analysis. It holds generally.

\section{Method}

To do an error analysis, an interval for each of the four dimensionless parameters (10) must be specified to define the domain of definition. Taking as the only sampling values the two interval limits for all four parameters results in $2^{4}=16$ parameter combinations. They correspond to the edge points of a cuboid in the four-dimensional parameter space. These combinations represent the absolute minimum set needed to guarantee that the error analysis tests all four dimensions of the parameter space. One can augment the number of sampling values in each interval step by step, and the resulting estimation of the maximum error will eventually converge.

All but one of the six formulae have turned out to be scalable (see below). This allowed to set the side length $A$ of the reference coils to some fixed standard value, namely

$$
A=1 \mathrm{~mm} \text {. }
$$

It is desirable to opt for as large intervals of the design parameters as possible to get a large domain of definition, thus 
maximizing the scope of the analysis. The lowest feasible value of the number of windings of a spiral coil is $N=2$. For its maximum, $N=20$ was chosen. This value seemed to be a reasonable upper limit for all main applications of planar spiral coils (inductors in RF IC's, short-wave RFID and telemetry antennas). So, we have $N \in[2,20] \cap \mathbb{N}$. As for the filling factor $\rho$, low values down to 0.01 are preferred in RFID transponder antennas. This is because the coil is wound in the form of a thin wire as close to the rim of the transponder card as permitted by the standard ISO 7810 to maximize the coupling between transponder and reader. In reader antennas, $\rho$ is also kept as small as possible for the same reason. The same is true for telemetry antennas. In RF IC's, however, coupling to an external device is not intended. Rather, the objective is to exploit the available area as far as possible, which means practically filling it with windings. This is equivalent to preferring large values of $\rho$ up to 1. But due to the validity condition (19), this idealized upper limit had to be lowered. For the parameters $\kappa$ and $\gamma$, the intervals $\kappa \in[1.1,10]$ and $\gamma \in[1,1000]$ were considered useful. Now the upper limit of $\rho$ according to equation (19) could be determined. The more stringent condition results for the largest value of $\kappa$, so $\kappa=10$ had to be taken. Hence, for $N=2$, this results in the interval $\rho \in$ $[0.01,0.36]$, and for $N=20, \rho \in[0.01,0.90]$.

The precise reference values of the inductances were calculated numerically with the help of the well-known numerical software FastHenry2 [4], which has become the scientific and industrial standard for inductance calculations for systems of conductors with rectangular cross section. It is available for free download at www.fastfieldsolvers.com.

A script in the free programming language python was written based on the inverse transformation equations (12) (15) to automatically produce the required input data file directly from the dimensionless parameters (10), and for automatically running FastHenry2 repeatedly for all parameter combinations. The resulting reference inductances were then imported into MATLAB ${ }^{\circledR}$ to analyze the accuracy of the formulae.

Table 1 presents the resulting minimum number of 16 parameter combinations and the respective precise inductances $L$, numbered in the first column (\#). The parameters are in the order of decreasing influence on the inductance, i.e. $N$ is the most and $\gamma$ the least influential parameter. The calculations were done at DC, requesting $2 \times 2$ subfilaments. For the conductivity of copper at $20^{\circ} \mathrm{C}$, the value $\sigma=5.9595 \cdot 10^{7} \Omega^{-1} \mathrm{~m}^{-1}$ was used.

Table 2 lists the same coil designs as Table 1, in the same order, but with the corresponding (approximate) values of the more familiar dimensional parameters.

To test the scalability of the formulae, the same 16 calculations were repeated for the two extreme side lengths $A=$ $10 \mu \mathrm{m}$ and $A=100 \mathrm{~mm}$. Perfect scalability means that the relative error of a formula does not depend on $A$. For all six formulae except the "monomial expression" [8], the inductances of all combinations for $A=10 \mu \mathrm{m}$ and $A=100 \mathrm{~mm}$ deviated by less than $0.02 \%$ from the one obtained for the standard length $A=1 \mathrm{~mm}$. For the "monomial expression", the maximum deviation was $10 \%$. So, all formulae but the "monomial expression" are considered as scalable.

Table 3 shows the relative deviations of the six formulae for all 16 parameter combinations from the reference inductances $L$. The formulae are presented and discussed in section 4. Note that for the deviations of Jenei's formula in the last column, the layout of Fig. 2 was used to calculate the precise reference inductances. Further, Jenei's formula was not evaluated in its original form as published by the authors [9], but by using equation (32) for the total conductor length. Otherwise, the minimum error would be $3 \%$, and the maximum error would be as large as $101 \%$ !

For each formula, the maximum error resulting from the deviations in Table 3 is compiled in Table 4.

For all six formulae, it was found that the domain of definition of Table 1 was too demanding. If one is not prepared to restrict the domain of definition, then one is forced to accept the large maximum errors exposed in Table 4! They are too large to be useful in circuit design. The domain of definition had to be restricted before any of the formulae could be considered as useful.

One way to restrict the domain of definition is by subdividing the interval of $N$ and combining the resulting subintervals with adapted subintervals of $\rho$, subject to the validity condition (19). The final subintervals of $\rho$ could only be determined after the subintervals of $N$ had been specified, since their upper limit depends on the lower limit of the respective subinterval of $N$, see the explanation following equations (20).

Table 1: The precise inductances $L$ for the 16 combinations of the dimensionless parameters $N, \rho, \kappa$, and $\gamma$, for $A=1 \mathrm{~mm}$ and the layout according to Fig. 1 .

\begin{tabular}{cccccc}
\hline$\#$ & $N$ & $\rho$ & $\kappa$ & $\gamma$ & $\begin{array}{c}L \\
{[\mathrm{nH}]}\end{array}$ \\
\hline 1 & 2 & 0.01 & 1.1 & 1 & 15.676 \\
2 & 2 & 0.01 & 1.1 & 1000 & 16.924 \\
3 & 2 & 0.01 & 10 & 1 & 17.365 \\
4 & 2 & 0.01 & 10 & 1000 & 18.465 \\
5 & 2 & 0.36 & 1.1 & 1 & 3.8140 \\
6 & 2 & 0.36 & 1.1 & 1000 & 4.7758 \\
7 & 2 & 0.36 & 10 & 1 & 4.7201 \\
8 & 2 & 0.36 & 10 & 1000 & 5.5240 \\
9 & 20 & 0.01 & 1.1 & 1 & 1674.5 \\
10 & 20 & 0.01 & 1.1 & 1000 & 1689.3 \\
11 & 20 & 0.01 & 10 & 1 & 1695.7 \\
12 & 20 & 0.01 & 10 & 1000 & 1706.8 \\
13 & 20 & 0.9 & 1.1 & 1 & 180.75 \\
14 & 20 & 0.9 & 1.1 & 1000 & 188.51 \\
15 & 20 & 0.9 & 10 & 1 & 191.65 \\
16 & 20 & 0.9 & 10 & 1000 & 197.58 \\
\hline
\end{tabular}

The first runs of the python script on the way to reducing the domain of definition were done with the minimum set of 16 sampling values. It was found to be useful to split the interval of $N$ into four parts. They were named after the respective ranges of $N$ : 
Two-windings range: $N=2$.

Low-range: $N \in[3,7] \cap \mathbb{N}$.

Mid-range: $N \in[8,12] \cap \mathbb{N}$.

High-range: $N \in[13,20] \cap \mathbb{N}$.

Table 2: Same as Table 1, but with the approximate values of the corresponding dimensional parameters, numbered in the same order.

\begin{tabular}{cccccc}
\hline$\#$ & $N$ & $\begin{array}{c}w \\
{[\mu \mathrm{m}]}\end{array}$ & $\begin{array}{c}s \\
{[\mu \mathrm{m}]}\end{array}$ & $h$ & $\begin{array}{c}L \\
{[\mathrm{nH}]}\end{array}$ \\
\hline 1 & 2 & 5.211 & 4.737 & $4.74 \mu \mathrm{m}$ & 15.673 \\
2 & 2 & 5.211 & 4.737 & $4.74 \mathrm{~nm}$ & 16.962 \\
3 & 2 & 9.009 & 0.901 & $0.90 \mu \mathrm{m}$ & 17.365 \\
4 & 2 & 9.009 & 0.901 & $0.90 \mathrm{~nm}$ & 18.465 \\
5 & 2 & 158.7 & 144.2 & $144 \mu \mathrm{m}$ & 3.8354 \\
6 & 2 & 158.7 & 144.2 & $144 \mathrm{~nm}$ & 4.8306 \\
7 & 2 & 246.6 & 24.66 & $24.7 \mu \mathrm{m}$ & 4.7201 \\
8 & 2 & 246.6 & 24.66 & $24.7 \mathrm{~nm}$ & 5.5240 \\
9 & 20 & 0.498 & 0.452 & $0.45 \mu \mathrm{m}$ & 1674.5 \\
10 & 20 & 0.498 & 0.452 & $0.45 \mathrm{~nm}$ & 1689.3 \\
11 & 20 & 0.518 & 0.052 & $0.05 \mu \mathrm{m}$ & 1695.7 \\
12 & 20 & 0.518 & 0.052 & $0.05 \mathrm{~nm}$ & 1706.8 \\
13 & 20 & 24.32 & 22.11 & $22.1 \mu \mathrm{m}$ & 180.75 \\
14 & 20 & 24.32 & 22.11 & $22.1 \mathrm{~nm}$ & 188.51 \\
15 & 20 & 24.86 & 2.486 & $2.49 \mu \mathrm{m}$ & 191.65 \\
16 & 20 & 24.86 & 2.486 & $2.49 \mathrm{~nm}$ & 197.58 \\
\hline
\end{tabular}

Table 3: Percentaged deviations of the six formulae for the parameter combinations in Tables 1 and 2, numbered in the same order, from the precise inductances $L$.

\begin{tabular}{ccccccc}
\hline$\#$ & {$[6]$} & {$[7]$} & {$[8], 1$} & {$[8], 2$} & {$[8], 3$} & {$[9]$} \\
& {$[\%]$} & {$[\%]$} & {$[\%]$} & {$[\%]$} & {$[\%]$} & {$[\%]$} \\
\hline 1 & 51 & -19 & -27 & 8.1 & 5.3 & 0.0 \\
2 & 40 & -25 & -32 & 0.1 & -2.4 & -0.9 \\
3 & 37 & -15 & -35 & -2.8 & 11 & 0.0 \\
4 & 29 & -20 & -39 & -8.6 & 4.3 & -0.1 \\
5 & 1.4 & 33 & 30 & 29 & 35 & -0.9 \\
6 & -19 & 6.5 & 4.1 & 3.0 & 8.0 & -1.7 \\
7 & -7.2 & -7.1 & -5.7 & -6.7 & 14 & 1.4 \\
8 & -21 & -21 & -19 & -20 & -2.2 & 1.1 \\
9 & 44 & -6.1 & -32 & 0.7 & -10 & -5.0 \\
10 & 43 & -6.9 & -33 & -0.2 & -11 & -5.2 \\
11 & 69 & 15 & -33 & -0.6 & 13 & -4.9 \\
12 & 68 & 14 & -34 & -1.2 & 13 & -4.9 \\
13 & 22 & 11 & 0.7 & 4.5 & -6.1 & -25 \\
14 & 17 & 6.0 & -3.4 & 0.2 & -10 & -25 \\
15 & 43 & 25 & -6.8 & -3.3 & 11 & -23 \\
16 & 38 & 21 & -9.6 & -6.2 & 8.0 & -23 \\
\hline
\end{tabular}

This subdivision allowed to assess the accuracy in each of the resulting subdomains separately. This was useful because they could be made to correlate with the main areas of application of planar spiral coils. In the case $N=2$, Jenei's formula [9] produced very low errors (Table 3, \# 1 - 8), so it was considered worthwhile to single this case out.

Table 4: Maximum errors from Table 3.

\begin{tabular}{lc}
\hline \multicolumn{1}{c}{ Formula } & Max. error [\%] \\
\hline Crols [6] & 69 \\
Ronkainen [7] & 33 \\
Mohan [8], 1, modified Wheeler & 39 \\
Mohan [8], 2, Current sheet approx. & 29 \\
Mohan [8], 3, Monomial expression & 35 \\
Jenei [9] & 25 \\
\hline
\end{tabular}

The upper limit of the low-range interval was chosen to be 7 because, to our knowledge, in the 26 years' history of LEGIC Identsystems ${ }^{\circledR}$ AG, none of the company's over 250 licensees ever produced an antenna for a transponder card or a reader consisting of a coil with more than 7 windings. So, the first two intervals were thus made to belong to subdomains that mainly represent RFID antennas and, since in telemetry systems, RFID transponder chips are often used [3], also telemetry antennas. The upper limit 12 of the midrange interval was chosen somehow arbitrarily. The midand high-range intervals belong to subdomains that mainly cover inductors in RF IC's because the validity condition (19) allows these intervals to be combined with large $\rho$ (see below), and large $\rho$ are typical for inductors in RF IC's, as explained above.

Now that the subintervals of $N$ were fixed, the adapted subintervals of $\rho$ could be specified. As before, for $N=2$ one finds $\rho \in[0.01,0.36]$. If $\rho$ lies within this interval, then the validity condition is always met. Analogously, the intervals for the other ranges were found by evaluating equation (19) for $\kappa=10$ and for the respective lower limit of $N$ :

Two-windings range: $N=2, \rho \in[0.01,0.36]$.

Low-range: $N \in[3,7] \cap \mathbb{N}, \rho \in[0.01,0.52]$.

Mid-range: $N \in[8,12] \cap \mathbb{N}, \rho \in[0.01,0.78]$.

High-range: $N \in[13,20] \cap \mathbb{N}, \rho \in[0.01,0.86]$.

For all ranges: $\kappa \in[1.1,10], \gamma \in[1,1000]$.

The runs of the python script were then repeated by inserting more and more intermediate sampling values of $\rho$ and $\kappa$ into the center between the ones that had already been present in the previous run, and for all four ranges as defined above. For the parameter $\gamma$, logarithmically equally spaced sampling values were inserted because of the large span of its interval. This resulted in numbers of initially 2 , then 3 , 5 , and finally 9 sampling values of the parameters $\rho, \kappa$, and $\gamma$ in subsequent runs. The number of values of $N$ had to be adapted to the number available in the discrete interval of the respective range. After each run, the new maximum errors were compared with those from the previous one. After the fourth run, all maximum errors remained the same to four digits after the decimal point, except that in the midrange, Ronkainen's increased by $1.0 \%$, and there were 
three other increases of $0.06 \%$ maximum, and in the highrange, Ronkainen's increased by $1.5 \%$, and there were two other growths of $0.07 \%$ and $0.02 \%$. For practical purposes, the maximum errors were then regarded as having converged. The sampling values of the last run are listed below.

Two-windings range: $N=2$,

$\rho=0.01,0.0537,0.0975,0.1412,0.1850,0.2288,0.2725$, $0.3162,0.36$.

Low-range: $N=3,4,5,6,7$, $\rho=0.01,0.0737,0.1375,0.2013,0.2650,0.3287,0.3925$, $0.4563,0.52$.

Mid-range: $N=8,9,10,11,12$,

$\rho=0.01,0.1063,0.2025,0.2988,0.3950,0.4913,0.5875$, $0.6838,0.78$.

High-range: $N=13,14,15,16,17,18,19,20$, $\rho=0.01,0.1162,0.2225,0.3287,0.4350,0.5413,0.6475$, $0.7538,0.86$.

For all ranges: $\kappa=1.1,2.2125,3.3250,4.4375,5.5500,6.6625,7.7750$, $8.8875,10$.

$\gamma=1,2.4,5.6,13.3,31.6,75,177.8,422,1000$.

The calculations based on the above values comprised 1 . $9^{3}=729$ parameter combinations or reference designs in the two-windings range ( 1 value of $N, 9$ values each of $\rho$, $\kappa$, and $\gamma$ ), plus $5 \cdot 9^{3}=3645$ each in the low- and midrange ( 5 values of $N$ ), plus $8 \cdot 9^{3}=5832$ in the high-range ( 8 values of $N$ ), totaling 13'851 parameter combinations.

With the four disjunct subdomains identified by the four ranges of $N$ as defined above, the maximum errors of two of the formulae had at least decreased enough for them to be useful for circuit design in one area of application. The results can be found in the Tables of section 4 .

\section{Inductance formulae from the literature}

In this section, the six inductance formulae from the literature [6] - [9] are discussed in detail. The maximum errors shown in Tables $5-11$ were all calculated based on the $13^{\prime} 851$ parameter combinations obtained from the Cartesian products of the sets of sampling values listed at the end of section 3. In SI units, all formulae contain the magnetic permeability of the vacuum, $\mu_{0}=4 \pi \cdot 10^{-7} \mathrm{Vs} /(\mathrm{Am})$.

\subsection{Crols' formula}

\subsubsection{Definition and description}

Crols et al. presented an empirical formula ([6], Table 1). Unfortunately, the authors failed to give a derivation. With the definition of parameters introduced in section 2 , it reads

$$
L=1.3 \frac{\mu_{0}}{4 \pi} \cdot \frac{S_{t o t^{\frac{3}{2}}}}{s^{2}} \cdot\left(\frac{S_{m e t}}{S_{t o t}}\right)^{\frac{5}{3}} \cdot\left(\frac{s}{w}\right)^{\frac{1}{4}},
$$

where $S_{\text {tot }}=A_{o}{ }^{2}$ is the total coil surface, and $S_{m e t}$ is the surface of the coil covered by metal. The authors missed to give an equation for calculating $S_{m e t}$. It is derived by what follows as

$$
S_{m e t}=l \cdot s-4 N s^{2},
$$

where $l$ is the total conductor length. $S_{\text {met }}$ is approximately given by $l \cdot s$. But this way, in every corner of the windings, the small square area $s^{2}$ would be counted twice, so the product $l \cdot s$ needs to be corrected by this small area for every winding and in all four corners. This leads to equation (22).

Now, one still needs an equation for the total conductor length $l$. When the coil is constructed as illustrated in Fig.1, then $l$ is given by the expression

$$
l=4 N A_{i}+\left(4 N^{2}-4 N-1\right) w .
$$

Starting with the innermost horizontal conductor segment and spiraling outwards, the lengths of the four segments of the first winding are $A_{i}-w, A_{i}, A_{i}, A_{i}$. In total, for $N=1$, this gives $l=4 A_{i}-w$.

Proceeding with the second winding, the left vertical segment of the first winding is now elongated by $w$ to $A_{i}+w$. The lengths of the four adjacent segments forming the second winding are $A_{i}+w, A_{i}+2 w, A_{i}+2 w, A_{i}+2 w$. So, the lengths of all eight segments of the first two windings are $A_{i}-w, A_{i}, A_{i}, A_{i}+w, A_{i}+w, A_{i}+2 w, A_{i}+2 w$, $A_{i}+2 w$. Hence, for $N=2$, we have $l=8 A_{i}+7 w$.

Every winding contributes the length $4 A_{i}$ plus a correction in $w$ due to spiraling outwards. The correction factor of $w$ is given for all $N \in \mathbb{N}$ by the parenthesized polynomial in equation (23). The proof is given in the appendix.

\subsubsection{Results and discussion}

The results of the present study are shown in Table 5. The maximum errors are much larger than the overly optimistic value of $10 \%$ reported by the authors ([6], Fig. 2). They didn't provide any information concerning the domain of definition used in their error analysis. Thus, for any purpose other than estimating the error of the formula on the very same set of reference designs they used - which is known by themselves only - their statistics is of no use. The errors revealed in Table 5 are too large for the formula to be useful in circuit design.

Table 5: Results for the maximum errors of Crols' formula.

\begin{tabular}{cccc}
\hline $\begin{array}{c}\text { Two wind. } \\
{[\%]}\end{array}$ & $\begin{array}{c}\text { Low-range } \\
{[\%]}\end{array}$ & $\begin{array}{c}\text { Mid-range } \\
{[\%]}\end{array}$ & $\begin{array}{c}\text { High-range } \\
{[\%]}\end{array}$ \\
\hline 51 & 63 & 67 & 69 \\
\hline
\end{tabular}




\subsection{Ronkainen's formula}

\subsubsection{Definition and description}

The next empirical formula discussed in this paper was suggested by Ronkainen et al. ([7], equation (1)). Unfortunately, they didn't explain how they found it. It is given by

$$
L=1.5 \mu_{0} N^{2} A e^{-\frac{3.7(N-1) w}{A}} \cdot\left(\frac{A}{S}\right)^{0.1}
$$

\subsubsection{Results and discussion}

The authors presented comparisons to measurements ([7], Fig. 7). They included just one series of designs, in which they varied the number of turns only. Hence, these statistics must miss the maximum error. It comes as no surprise that their value reported as $5 \%$ has turned out to be by far too optimistic. The results of the present study are shown in Table 6. Although in all four subdomains of definition, the formula offers a clear improvement over Crols', the errors are still too large for equation (24) to be used in circuit design.

Table 6: The maximum errors of Ronkainen's formula.

\begin{tabular}{cccc}
\hline $\begin{array}{c}\text { Two wind. } \\
{[\%]}\end{array}$ & $\begin{array}{c}\text { Low-range } \\
{[\%]}\end{array}$ & $\begin{array}{c}\text { Mid-range } \\
{[\%]}\end{array}$ & $\begin{array}{c}\text { High-range } \\
{[\%]}\end{array}$ \\
\hline 33 & 23 & 23 & 30 \\
\hline
\end{tabular}

\subsection{Mohan's "modified Wheeler formula"}

\subsubsection{Definition and description}

Mohan et al. presented three formulae. The first one they named "modified Wheeler formula" ([8], equation (1)). As the name suggests, they obtained it by modifying one of Wheeler's empirical formulae [11]. Unfortunately, they did not explain how they arrived at their modification. The result is defined by the expression

$$
L=2.34 \mu_{0} \frac{N^{2} a}{1+2.75 \rho},
$$

where $a$ is the average side length, i.e. the mean value of the outermost side length $A_{o}$ and the innermost one $A_{i i}$ or, identically, the mean value of $A$ and $A_{i}$. With the help of equations (1) - (3), it can be expressed as

$$
a=A-(N-1) w .
$$

\subsubsection{Results and discussion}

The author's comprehensive Table of 61 coil designs including measured values of the respective inductances ([8], Table IV, $10^{\text {th }}$ column, \#52) disclosed a maximum error of $19.9 \%$. The results of the present study are given in Table 7. The errors of the "modified Wheeler formula" are larger than those of Ronkainen's, thus not warranting the use of the former in circuit design either.

Table 7: The maximum errors of Mohan's "modified Wheeler formula".

\begin{tabular}{cccc}
\hline $\begin{array}{c}\text { Two wind. } \\
{[\%]}\end{array}$ & $\begin{array}{c}\text { Low-range } \\
{[\%]}\end{array}$ & $\begin{array}{c}\text { Mid-range } \\
{[\%]}\end{array}$ & $\begin{array}{c}\text { High-range } \\
{[\%]}\end{array}$ \\
\hline 39 & 37 & 35 & 34 \\
\hline
\end{tabular}

\subsection{Mohan's current sheet approximation}

\subsubsection{Definition and description}

Mohan et al.'s second formula ([8], equation (2)) resulted from physical principles, namely, from a current sheet approximation. It reads

$$
L=c_{1} \mu_{0} N^{2} a\left[\log \left(\frac{c_{2}}{\rho}\right)+c_{3} \rho+c_{4} \rho^{2}\right]
$$

where $a$ is given by equation (26), and log is the natural logarithm. Despite its physical basis, the formula still contains four fitting constants, namely $c_{1}=0.635, c_{2}=2.07$, $c_{3}=0.18$, and $c_{4}=0.13$. The authors gave a very brief derivation in textual form only. They didn't mention how and with what data they fitted the constants $c_{i}, i=1 \ldots 4$, but one can assume that the same data as for the "monomial expression" was used, see paragraph 4.5.1.

\subsubsection{Results and discussion}

From the comprehensive Table of 61 coil designs including measured inductances presented by the authors, a maximum error of $19.5 \%$ could be inferred ([8], Table IV, $11^{\text {th }}$ column, \#52). The authors' value fits in well somewhere between the low- and mid-range of the present study, closer to the former, see Table 8. Equation (27) offers the lowest maximum errors of all six inductance formulae in the midand high-range. This makes the formula the best choice for designing inductors in RF IC's. The maximum errors are reasonably low for the formula to be useful for calculations where moderate precision suffices.

Table 8: The maximum errors of Mohan's current sheet approximation.

\begin{tabular}{cccc}
\hline $\begin{array}{c}\text { Two wind. } \\
{[\%]}\end{array}$ & $\begin{array}{c}\text { Low-range } \\
{[\%]}\end{array}$ & $\begin{array}{c}\text { Mid-range } \\
{[\%]}\end{array}$ & $\begin{array}{c}\text { High-range } \\
{[\%]}\end{array}$ \\
\hline 29 & 23 & 13 & 8.8 \\
\hline
\end{tabular}

\subsection{Mohan's "monomial expression"}

\subsubsection{Definition and description}

Mohan et al.'s third formula is an empirical product of power functions of dimensional parameters. The authors called it a "monomial expression" ([8], equation (3) and Table III). Actually, in common usage, only a power function with non-negative integer exponent is termed a "monomial" [12]. The coefficient $1.62 \cdot 10^{-5}$ (in SI units) and 
the five exponents of the power functions were reportedly obtained by solving a system of linear equations as a leastsquares fit to the logarithm of $19^{\prime} 000$ numerically obtained inductances. With the parameters in our notation as defined in section 2 and with all parameters in SI units, the formula reads

$$
\begin{gathered}
L=1.62 \cdot 10^{-5} \frac{\mu_{0}}{4 \pi}\left(A_{o} \cdot 10^{6}\right)^{-1.21} \cdot\left(s \cdot 10^{6}\right)^{-0.147} \\
\cdot\left(a \cdot 10^{6}\right)^{2.4} \cdot N^{1.78} \cdot\left(g \cdot 10^{6}\right)^{-0.03}
\end{gathered}
$$

where $a$ is given by equation (26). In section 2 it was shown that coil inductance scales linearly with the side length $A$ if all physical dimensions are stretched by the same factor. Hence, this must also apply to an inductance formula if it is to be scalable. Equations (25) and (27) are good examples of scalable formulae: Their result is proportional to $a$, which, by equations (13) and (26), is proportional to $A$, but $\rho$ remains constant upon stretching because it is a ratio of size-dependent parameters, see its definition (6). By contrast, equation (28) does not scale linearly with the side length, neither with $A_{o}$ nor with $a$, nor in the combination of all its parameters. Therefore, the formula cannot be scalable, as was observed in section 3 . It seems odd that two parameters related to the side length, namely, $A_{o}$ and $a$, were used, instead of just one, and instead of taking the filling factor $\rho$ as a further parameter, as the authors had done in equations (25) and (27).

\subsubsection{Results and discussion}

The data given by the authors revealed a maximum error of $18.5 \%$ ([8], Table IV, $12^{\text {th }}$ column, \#52). This comes quite close to the results for the mid- and high-range of the present study, see Table 9. Clearly, the "monomial expression" is less accurate than the current sheet approximation, see Table 8. This disproves Jenei et al.'s assertion that “... the monomial formula is the most accurate closed-form expression published up to date" ([9], p. 79) in referencing Mohan et al. [8]. This statement appears even odder when one considers that Jenei et al. emphasized the importance of scalability ([9], p. 77) since the "monomial expression" is not scalable, see section 3 and paragraph 4.5.1, whereas the current sheet approximation, which appeared in the same paper [8], is scalable. The maximum errors of the "monomial expression" are too large for it to be useful in circuit design.

Table 9: The maximum errors of Mohan's "monomial expression".

\begin{tabular}{cccc}
\hline $\begin{array}{c}\text { Two wind. } \\
{[\%]}\end{array}$ & $\begin{array}{c}\text { Low-range } \\
{[\%]}\end{array}$ & $\begin{array}{c}\text { Mid-range } \\
{[\%]}\end{array}$ & $\begin{array}{c}\text { High-range } \\
{[\%]}\end{array}$ \\
\hline 35 & 26 & 22 & 22 \\
\hline
\end{tabular}

\subsection{Jenei's formula}

\subsubsection{Definition and description}

Jenei et al. developed a formula ([9], equation (7)) from physical principles, representing an approximation to the
Greenhouse method [5]. They derived approximate analytical expressions for the total inductance of all constituent conductor segments and for the average mutual inductances between segments of an average length at an average distance instead of summing up all the exact contributions. Unfortunately, they didn't disclose the derivations of the total conductor length $l$ and the average distance $d^{+}$between segments on the same side of the square. These expressions allowed them to set up an analytical formula for the inductance as a function of the design parameters:

$$
\begin{aligned}
& L=\frac{\mu_{0} l}{2 \pi}\left\{\log \left(\frac{l}{N(s+h)}\right)-0.2-0.47 N+(N-1)\right. \\
& \cdot\left\{\log \left[\sqrt{1+\left(\frac{l}{4 N d^{+}}\right)^{2}}+\frac{l}{4 N d^{+}}\right]\right. \\
&\left.\left.-\sqrt{1+\left(\frac{4 N d^{+}}{l}\right)^{2}}+\frac{4 N d^{+}}{l}\right\}\right\} .
\end{aligned}
$$

The constants 0.2 and 0.47 are approximations of constants arising in the physical derivation; they are no fitting constants. The length $l$ is the total length of all conductor segments, and log denotes the natural logarithm. For integer number of turns, the expression given for $l$ by the authors ([9], equation (1)) reduces in the present notation to

$$
l=(4 N+1)\left(A_{i}+N w\right)
$$

Note that this equation is false. The correct equation for the total conductor length $l$ of a square planar spiral coil was derived in equation (23). Equation (30) grossly overestimates the total length. This may lead to large errors in the inductance, see the comment to Table 3, or Table 11.

One might argue that Jenei et al.'s derivation was based on a different coil layout than the one shown in Fig. 1. This was indeed the case, see Fig. 2 (based on [9], Fig. 1).

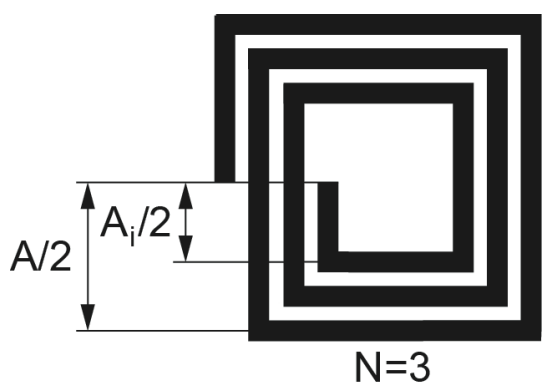

Figure 2: The layout of a square planar spiral coil of three windings $(N=3)$ used by Jenei et al. [9].

The total conductor length loses $A / 2$ compared to Fig. 1 but gains $A_{i} / 2$ in return. Since the latter is smaller than the former, for an otherwise identical coil, the net total length decreases. Hence, equation (30) overestimates the length 
even more. The correct formula for the total conductor length $l_{2}$ for the layout of Fig. 2 can be expressed in terms of $l_{1}$, the conductor length of the layout of Fig. 1, which was derived in equation (23). By what was said above, the expression is

$$
l_{2}=l_{1}-\frac{A}{2}+\frac{A_{i}}{2}
$$

With the help of equations (2) and (23), this becomes

$$
l_{2}=4 N A_{i}+\left(4 N^{2}-5 N\right) w .
$$

Consequently, in this study, equation (32) was used for $l$ in equation (29).

For estimating the (positive) mutual inductance between adjacent conductor segments of equal current direction, the authors gave a mean distance $d^{+}([9]$, equation (5)), which occurs in equation (29). For integer number of turns, it reads

$$
d^{+}=\frac{(N+1)}{3} w
$$

It is to be expected that an approximation like equation (29), which is based on the replacement of interactions between many segments of different lengths and distances by an average interaction between two segments of equal length at an average distance, will be the more accurate the smaller the variance among the real interactions. This variance increases with increasing $N$ and $\rho$, so that the approximation is expected to be most precise for low $N$ and low $\rho$.

\subsubsection{Results and discussion}

The authors gave comparisons to measurements. They presented a statistical error distribution ([9], Fig. 4), according to which the maximum error for a set of 20 inductors was $8 \%$, and the same result was obtained independently for a set of 7 inductors from the literature ([9], Fig. 5). The domains of definition were not specified. So, as was argued for the same situation in paragraph 4.1.2, such statistics is of very limited use. The small sets of reference designs were certainly not able to homogeneously scan the parameter space in all its dimensions.

Table 10 shows the results of the present study. Equation (29) features the smallest maximum errors of all six inductance formulae in the two-windings and low-range, but only if the correct equation (32) for the total length of the conductor is used instead of the original equation (30) published by the authors. Especially the maximum error of $1.7 \%$ in the two-windings range is very low. For more than two windings, Table 10 discloses a maximum error of $9.3 \%$. Since most transponder and reader coils have 4 windings, it may be worthwhile to mention that under the restriction of $N \leq 4$ and $\rho \leq 0.2$ (to our knowledge, none of the licensees of LEGIC Identsystems AG ever produced an antenna for a transponder card or a reader consisting of a coil with $\rho>0.15$ ), the present error analysis revealed a maximum error of $3.9 \%$. Clearly, equation (29) is the formula of choice for designing antennas for RFID and telemetry systems.

Table 10 nicely confirms the theoretical expectation that equation (29) should be most accurate for low $N$ and low $\rho$ as stated in paragraph 4.6.1. In Table 10, the higher the range, the larger the error. In the mid- and high-range, the formula cannot compete with equation (27), see Table 8 .

Concerning the effect of the coil layout discussed in paragraph 4.6.1, all 13'851 calculations were repeated with the modified layout according to Fig. 2. The effect of the layout on the inductance was found to be $8.9 \%$ maximum in the two-windings range and $2.7 \%$ maximum in the high-range. One expects the maximum errors of equation (29) to decrease if the correct layout is used. This was indeed the case in all ranges.

Tables 3, 4, and 10 contain the results for equation (29) obtained with the correct layout according to Fig. 2 and with $l$ evaluated with equation (32).

Table 10: The maximum errors of Jenei's formula for the layout shown in Fig. 2, with $l$ evaluated according to equation (32).

\begin{tabular}{cccc}
$\begin{array}{c}\text { Two wind. } \\
{[\%]}\end{array}$ & $\begin{array}{c}\text { Low-range } \\
{[\%]}\end{array}$ & $\begin{array}{c}\text { Mid-range } \\
{[\%]}\end{array}$ & $\begin{array}{c}\text { High-range } \\
{[\%]}\end{array}$ \\
\hline 1.7 & 9.3 & 18 & 24 \\
\hline
\end{tabular}

Table 11 shows the results for the same layout but with $l$ evaluated with Jenei et al.'s original but erroneous equation (30). By chance, the maximum error in the high-range is smaller in Table 11 than it is in Table 10, yet larger than the one in Table 8. But the maximum errors in the twowindings and the low-range are extremely large.

Table 11: Same as Table 10, but with $l$ evaluated according to Jenei's original equation (30).

\begin{tabular}{cccc}
\hline $\begin{array}{c}\text { Two wind. } \\
{[\%]}\end{array}$ & $\begin{array}{c}\text { Low-range } \\
{[\%]}\end{array}$ & $\begin{array}{c}\text { Mid-range } \\
{[\%]}\end{array}$ & $\begin{array}{c}\text { High-range } \\
{[\%]}\end{array}$ \\
\hline 101 & 70 & 15 & 14 \\
\hline
\end{tabular}

\section{Conclusions}

Many analytical inductance formulae for square planar spiral coils (with rectangular conductor cross section) can be found in the literature. Concerning the accuracy of a formula, for the design engineer, only the knowledge of its maximum error over a specified domain of definition is meaningful. In this paper, all the parameters used to describe square planar spiral coils have been exposed, even if redundant, as has been necessary to discuss the various formulae from the literature in a consistent way. Further, equations for transforming dimensional into dimensionless design parameters and vice versa, have been derived. It has been shown that the transition from dimensional to dimensionless parameters allows to reduce the dimensionality of the parameter space from five to four. Further, two equations for testing a given combination of parameter values on its geometric validity 
have been derived, one for dimensional parameters, and the other for dimensionless ones.

The maximum errors of six of some of the most cited formulae in the literature have been compared over four specified domains of definition, each of which scans all four dimensions of the parameter space. To arrive at a sufficient convergence of all maximum errors, it has been necessary to consider more than $13^{\prime} 000$ reference designs, whose precise inductance has been computed numerically.

It has been found that the error statistics published in many papers is unreliable. Some authors markedly overestimated the accuracy of their formula, likely due to failing to choose a set of reference designs that tests all dimensions of the parameter space.

Mohan et al.'s current sheet approximation, equation (27), has been found to be the most accurate one for designing inductors in RF IC's, whereas Jenei et al.'s formula, equation (29), has turned out to be the best choice for designing RFID and telemetry antennas, provided the correct equation for the total length of the conductor derived in this paper, equation (32), is used.

It is suggested that Mohan et al.'s "monomial expression" might be improved by using dimensionless parameters instead of dimensional ones, and only one size parameter instead of two, but as a linear factor to make the formula scalable. This way, the number of dimensions could be reduced from five to four and, hence, the number of fitting constants from six to five.

Further research in finding better formulae is highly encouraged. A generally applicable formula with good accuracy has not been found yet, despite the claims to the contrary made by some of the authors.

The method used in the present study for the error analysis may also prove useful for standardized error tests on future formulae. Using the same method, domains of definition, and sampling values as presented in section 3 will guarantee that only geometrically valid designs are considered, that all parameter space dimensions are scanned homogeneously, and that the results will be comparable to those reported in section 4 of this paper.

\section{Appendix}

Proof of the parenthesized correction factor in equation (23) by mathematical induction:

1. For $N=1$, the polynomial yields a correction factor of -1 . This is correct, see paragraph 4.1.1.

2. Assume that for $N=n$, the correction factor is given by $4 n^{2}-4 n-1$.

For $N=n+1$, the last segment of the last wind$\operatorname{ing} N=n$ on spiraling outwards, i.e. its leftmost vertical segment, is elongated by $w$, see Fig. 1 and paragraph 4.1.1. The length of the lower horizontal segment of the new winding is $A_{i}+(2 n-1) w$. The lengths of its remaining three segments are all $A_{i}+2 n \mathrm{w}$. In total, the correction factor increases by $1+2 n-1+3 \cdot 2 n=8 n$.
Now we have $4 n^{2}-4 n-1+8 n=4 n^{2}+4 n-$ $1=4(n+1)^{2}-4(n+1)-1$, q.e.d.

\section{Acknowledgements}

The author wishes to thank Mr. Hansruedi Waelle from LEGIC Identsystems AG for writing and installing the python script for the automated and repeated execution of the software FastHenry2.

\section{References}

[1] J. Chen and J. J. Liou, On-Chip Spiral Inductors for RF Applications: An Overview, Journal of Semiconductor Technology and Science, vol. 4, no. 3, 149-167, 2004.

[2] K. Finkenzeller, RFID Handbook: Fundamentals and Applications in Contactless Smart Cards, Radio Frequency Identification and Near-Field Communication, John Wiley \& Sons, Ltd, West Sussex, $3^{\text {rd }}$ ed., 2010.

[3] R. A. Potyrailo, C. Surman, S. Go, Y. Lee, T. Sivavec, and W. G. Morris, Development of radio-frequency identification sensors based on organic electronic sensing materials for selective detection of toxic vapors, Journal of Applied Physics, 106, 124902-1 - 124902-6, 2009.

[4] M. Kamon, M. J. Tsuk, and J. K. White, FASTHENRY: A Multipole-Accelerated 3-D Inductance Extraction Program, IEEE Trans. on Microwave Theory and Techniques, vol. 42, no. 9, 1750-1758, 1994.

[5] H. M. Greenhouse, Design of Planar Rectangular Microelectronic Inductors, IEEE Trans. on Parts, Hybrids, and Packaging, vol. 10, no. 2, 101-109, 1974.

[6] J. Crols, P. Kinget, J. Craninckx, and M. Steyaert, An Analytical Model of Planar Inductors on Lowly Doped Silicon Substrates for High Frequency Analog Design up to $3 \mathrm{GHz}$, IEEE Symposium on VLSI Circuits, Honolulu, Digest of Technical Papers, 28-29, 1996.

[7] H. Ronkainen, H. Kattelus, E. Tarvainen, T. Riihisaari, M. Andersson, and P. Kuivalainen, IC compatible planar inductors on silicon, IEE Proc. Circuits Devices Syst., vol. 144, no. 1, 29-35, 1997.

[8] S. S. Mohan, M. del Mar Hershenson, S. P. Boyd, and T. H. Lee, Simple Accurate Expressions for Planar Spiral Inductances, IEEE Journal of Solid-State Circuits, vol. 34, no. 10, 1419-1424, 1999.

[9] S. Jenei, B. K. J. C. Nauwelaers, and S. Decoutere, Physics-Based Closed-Form Inductance Expression for Compact Modeling of Integrated Spiral Inductors, IEEE Journal of Solid-State Circuits, vol. 37, no. 1, 77$80,2002$.

[10] H. A. Aebischer and B. Aebischer, Improved Formulae for the Inductance of Straight Wires, Advanced Electromagnetics, vol. 3, no. 1, 31-43, 2014. DOI: http://dx.doi.org/10.7716/aem.v3i1.254 
[11] H. A. Wheeler, Simple inductance formulas for radio coils, Proc. of the Institute of Radio Engineers, vol. 16, no. 10,1398 - 1400, 1928.

[12] https://en.wikipedia.org/wiki/Monomial 